\title{
Chemical Synthesis of Polysaccharides I. Synthetic Polysaccharide Having One Hydroxyl Group in Its Repeating Unit, 3,4-Dideoxy-(1 $\rightarrow 6)-\alpha-$ DL-erythro-hexopyranan
}

\author{
Masahiko OKADA, Hiroshi Sumitomo, and Ken Ogasawara \\ Faculty of Agriculture, Nagoya University, \\ Chikusa-ku, Nagoya 464, Japan
}

(Received June 7, 1982)

\begin{abstract}
A new DL-polysaccharide containing one hydroxyl group in its repeating unit, 3,4-dideoxy-( $1 \rightarrow 6)$ - $\alpha$-DL-erythro-hexopyranan, was synthesized by the cationic ring-opening polymerization of $4(a)$-benzyloxy-6,8-dioxabicyclo[3.2.1]octane (1), followed by debenzylation. The bicyclic acetal 1 was prepared from 3,4-dihydro-2 $H$-pyran-2-carbaldehyde via six step reactions. Ring-opening polymerization of $\mathbf{1}$ was initiated by antimony pentafluoride, antimony pentachloride, and trifluoromethanesulfonic acid to give polyacetal (2) with number average molecular weights of $6700-13500$ at $-60^{\circ} \mathrm{C}$ or below. Boron trifluoride etherate, a highly effective initiator for stereospecific polymerization of 6,8-dioxabicyclo[3.2.1]octane, was practically ineffective in initiating the polymerization of 1 . Debenzylation of 2 with sodium metal in liquid ammonia afforded a white powdery polymer identified as 3,4-dideoxy-(1 $\rightarrow 6)$ - $\alpha$-DL-erythro-hexopyranan (3) by ${ }^{13} \mathrm{C}$ NMR spectroscopy. The synthetic polysaccharide 3 having number average molecular weights of $4300-7800$ was soluble in dimethyl sulfoxide, $N, N$-dimethylformamide, and methanol, and insoluble in other common organic solvents and water. It melted at $130-140^{\circ} \mathrm{C}$ and decomposed in air at $280-300^{\circ} \mathrm{C}$. Water sorption of 3 was measured at different relative humidities and compared with that of natural dextran having an analogous structure.

KEY WORDS Synthetic Polysaccharide / Ring-Opening Polymerization /

Bicyclic Acetal / Water Sorption /
\end{abstract}

In recent years, many attempts have been made to synthesize structurally well-defined polysaccharides and their analogues, aiming at obtaining model polymers that would elucidate the sophisticated functions of naturally occurring polysaccharides in relation to molecular structure as well as new polymeric materials for potential biomedical utility. ${ }^{1-5}$ Most of the work done so far in this field has used carbohydrates as starting materials. In contrast, we recently synthesized several dextrantype polymers having hydroxyl, amino, and sulfate groups starting from non-carbohydrate sources: ${ }^{6-9}$ These polymers were obtained by appropriate chemical modifications of a polyacetal containing dihydropyran rings, poly(5,6-dihydro- $2 \mathrm{H}$-pyran6,2-diyloxymethylene), derived from 3,4-dihydro$2 \mathrm{H}$-pyran-2-carbaldehyde (acrolein dimer). Generally, this approach leads to optically inactive
DL-polysaccharides, but we have also devised a synthetic route for L-polysaccharides and their analogues from a similar starting material through optical resolution. ${ }^{10,11}$

However, this method has the drawback that epoxidation of the dihydropyran rings in the polymer takes place non-stereospecifically, thus resulting in structurally irregular polymers by subsequent chemical reactions of the epoxidized polymers. ${ }^{6,9}$ Therefore, we adopted an alternative synthetic procedure, namely, the synthesis of bicyclic acetals having appropriate substituents readily convertible to hydroxyl groups and their ring-opening polymerization. The present paper describes the chemical synthesis of a new DL-polysaccharide (3) having one hydroxyl group regiospecifically in its repeating unit by the ring-opening polymerization of $4(a)$ benzyloxy-6,8-dioxabicyclo[3.2.1]octane (1,6-an- 
hydro-2-O-benzyl-3,4-dideoxy-erythro-hexopyranose) (1) and subsequent debenzylation of the resulting polymer (2).

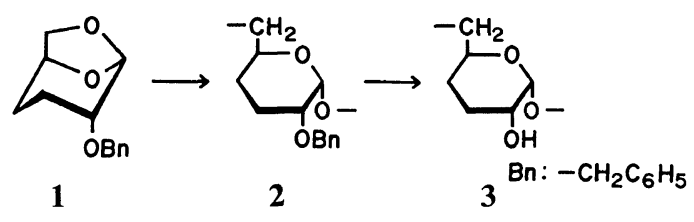

\section{EXPERIMENTAL}

4(a)-Benzyloxy-6,8-dioxabicyclo[3.2.1] octane (1) was prepared from 3,4-dihydro- $H$-pyran-2-carbaldehyde (acrolein dimer) (4) via six step reactions as shown in Scheme I. The precursor of 1, 4(a)hydroxy-6,8-dioxabicyclo[3.2.1]octane (9) was synthesized from 4 by the procedures described by Brown et al. ${ }^{12-14}$ with some modifications.

Preparation of 4(a)-Benzyloxy-6,8-dioxabicyclo[3.2.1] ]octane (1)

Sodium hydride $(1.0 \mathrm{~g}$ in $50 \%$ oil dispersion) was repeatedly washed with $n$-hexane to remove mineral oil, and dissolved in dry dimethyl sulfoxide $(10 \mathrm{ml})$. The solution was added to a solution of $4(a)$ hydroxy-6,8-dioxabicyclo[3.2.1]octane $\quad(2.6 \mathrm{~g}, 21$ $\mathrm{mmol}$ ) in the same solvent with vigorous stirring. The reaction mixture was stirred at room temperature for about $30 \mathrm{~min}$. Subsequently, freshly distilled benzyl chloride $(2.8 \mathrm{~g}, 22 \mathrm{mmol})$ was added dropwise to the mixture which was then heated at $60^{\circ} \mathrm{C}$ for $1 \mathrm{~h}$. After being cooled to room temperature, the reaction mixture was poured into a mixture of ice and water $(250 \mathrm{ml})$, followed by extraction with chloroform. The chloroform extract was washed repeatedly with water and dried over anhydrous magnesium sulfate. The solvent was removed by a rotary evaporator and the resulting viscous oil was chromatographed (column, silica gel: eluent, $n$-hexane-ethyl acetate $(7: 3, \mathrm{vol}))$ to provide 1 in a yield of $88 \%$. It was dried over calcium hydride for several days and distilled under reduced pressure before use. bp, $137-138^{\circ} \mathrm{C} / 1.0$ mmHg. Anal. Calcd. for $\mathrm{C}_{13} \mathrm{H}_{16} \mathrm{O}_{3}$ : C, $70.89 \%$; $\mathrm{H}, 7.33 \%$; Found: C, 71.27\%; H, 7.35\%. ${ }^{13} \mathrm{C}$ NMR ( $\left.\mathrm{CDCl}_{3}, \mathrm{TMS}\right), \delta 138.34$ phenyl (ipso), 128.25 phenyl (ortho), 127.52 phenyl (meta and para), $100.67 \mathrm{C}(5), 72.90 \mathrm{C}(4)$ and $\mathrm{C}(1), 71.14$ benzyl,
66.56 C(7), 25.14 C(2), and 20.17 ppm C(3).

\section{Polymerization of 4(a)-Benzyloxy-6,8-dioxabi- cyclo[3.2.1]octane (1) \\ Polymerization of 1 was carried out in methylene} chloride at different temperatures between -78 and $0^{\circ} \mathrm{C}$. Antimony pentafluoride, antimony pentachloride, trifluoromethanesulfonic acid, and boron trifluoride etherate were used as initiators. A monomer solution was charged into a glass ampoule and after being frozen in a liquid nitrogen bath, an initiator solution was added to the ampoule. The ampoule was then evacuated, sealed off, and kept in a constant temperature bath. After a prescribed period of time, a small amount of pyridine was added to the reaction mixture to terminate the polymerization. The mixture was poured into a large volume of methanol to precipitate a white powdery polymer. This polymer was purified by repeated reprecipitation using methylene chloride and methanol as a solvent and precipitant pair, and dried under reduced pressure at $50^{\circ} \mathrm{C}$.

\section{Debenzylation of Poly(3-benzyloxytetrahydropyran- 6,2-diyloxymethylene) (2)}

To liquid ammonia $(50 \mathrm{ml})$ in a three-necked flask equipped with a finger trap, there was added dropwise a solution of polymer $2(2.06 \mathrm{~g})$ dissolved in a mixture of toluene $(60 \mathrm{ml})$ and 1,2-dimethoxyethane $(20 \mathrm{ml})$ with external cooling with a dry icemethanol bath. The dry ice-methanol bath was removed, and small pieces of sodium metal $(0.56 \mathrm{~g})$ were added in portions to the solution until a dark blue color persisted. After stirring reaction mixture for $1 \mathrm{hr}$, a small amount of ammonium chloride was cautiously added to the mixture to decompose the excess sodium, followed by the dropwise addition of water $(15 \mathrm{ml})$. The finger trap was removed, and stirring was continued until most of the ammonia evaporated. The residual reaction mixture was centrifuged to separate precipitates which were then dissolved in methanol to remove dark-colored, insoluble impurities and reprecipitated by pouring the methanol solution into a large volume of water. The resulting white powdery polymer was dried under reduced pressure $t u$ a constant weight. Yield of the water insoluble polymer, $89 \% ; M_{n}=4.3 \times 10^{3}$.

The organic layer of the supernatant of the reaction mixture was separated from the aqueous layer and extracted with water several times. The 
water extract combined with the aqueous layer was extracted with methylene chloride and dialyzed in a stream of water for a few days. It was concentrated by a rotary evaporator and subsequently freezedried to yield a small amount of a white polymer. Yield of the water soluble polymer, $2 \%$.

\section{Characterization}

${ }^{1} \mathrm{H}$ and ${ }^{13} \mathrm{C}$ NMR spectra were recorded on JEOL MH-100 and JEOL FX-100 instruments working at 100 and $25 \mathrm{MHz}$, respectively. Chloroform- $d_{1}$ and benzene- $d_{6}$ were used for the benzylated polymer 2 , and methanol- $d_{4}$ and dimethyl sulfoxide- $d_{6}$ for the debenzylated polymer 3 . Tetramethylsilane was used as the internal reference. Number average molecular weights of the polymers were measured by a Hewlett Packard vapor pressure osmometer on solutions in benzene for 2 and in dimethylformamide for 3 at $37^{\circ} \mathrm{C}$.

\section{Measurement of Water Sorption}

Samples were dried to constant weight in a vacuum at $50^{\circ} \mathrm{C}$ prior to measuremnt. A sample was weighed in a phial and placed in a desiccator whose relative humidity was adjusted to a constant value by a saturated aqueous solution of appropriate inorganic salts. ${ }^{16}$ The desiccator was kept in a room controlled at $25^{\circ} \mathrm{C}$. Water sorption was represented as the percent of weight increase based on the dry samples.

\section{RESULTS AND DISCUSSION}

Synthesis of 4(a)-Benzyloxy-6,8-dioxabicyclo[3.2.1] octane (1)

4(a)-Benzyloxy-6,8-dioxabicyclo[3.2.1]octane (1,6-anhydro-2-O-benzyl-3,4-dideoxy- $\beta$-DL-ery- thro-hexopyranose) (1) was synthesized from 3,4-dihydro-2 $H$-pyran-2-carbaldehyde (4) via six step reactions as shown in Scheme I. The synthetic procedures for 4(a)-hydroxy-6,8-dioxabicyclo[3.2.1]octane (9) from 4 reported by Brown et $a l^{12-14}$ were employed with some modifications. Benzylation of 9 was successfully carried out using sodium hydride and benzyl chloride in dimethyl sulfoxide. The overall yield of $\mathbf{1}$ based on the starting material 4 was $11 \%$.

The bottleneck step for the synthesis of $\mathbf{1}$ is the dehydrobromination of 4-bromo-6,8-dioxabicyclo[3.2.1]octane (6) to 6,8-dioxabicyclo[3.2.1] oct-3ene (7). In this reaction, only $4(a)$-bromo-6,8-dioxabicyclo[3.2.1]octane is dehydrobrominated to. give 7, its 4(e)-counterpart remaining unreacted. Since the bromination of 2-hydroxymethyl-3,4dihydro- $2 H$-pyran (5) accompanied by cyclization gave a nearly equimolar stereoisomer mixture of $4(a)$ - and 4(e)-bromo-6,8-dioxabicyclo[3.2.1] loctane, $^{15}$ the yield of 7 in the subsequent dehydrobromination was generally below $50 \%$.

The epoxidation of 7 by $m$-chloroperbenzoic acid in methylene chloride followed by reduction with lithium aluminum hydride proceeds highly stereospecifically to give 9. ${ }^{12}$ Accroding to Brown et al., ${ }^{12}$ the epoxidation of 7 provides a stereoisomer mixture of $8(>95 \%)$ and $10(<5 \%)$. On lithium aluminum hydride reduction, $\mathbf{8}$ would give rise to 9 exclusively, since both polar and steric factor favor the attack of the reagent on the $\mathrm{C}(3)$ atom of 8 . In contrast, 10 would produce a mixture of 11 and $\mathbf{1 2}$, since a polar factor favors the attack of the $\mathrm{C}(3)$ atom of 10, while a steric factor favors the attack of the $\mathrm{C}(4)$ atom. ${ }^{17}$ (Numbering is based on the IUPAC nomenclature of organic chemistry.)
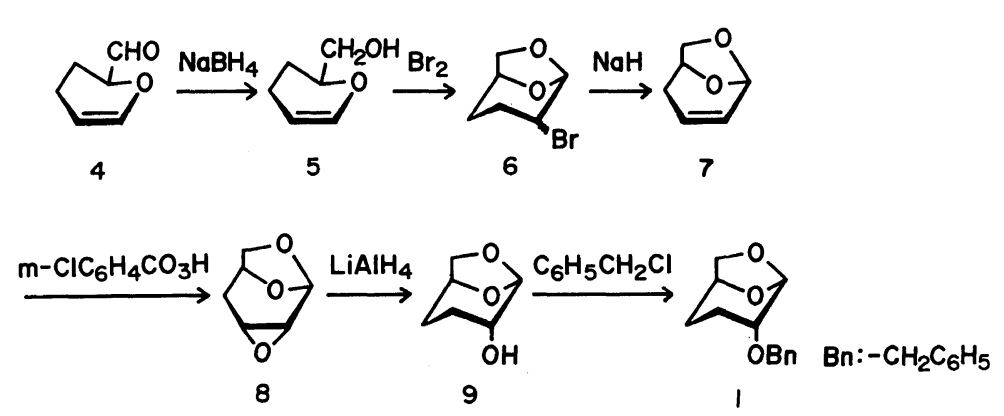

Scheme I. Synthetic route of $4(a)$-benzyloxy-6,8-dioxabicyclo[3.2.1]octane (1). 


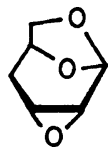

8
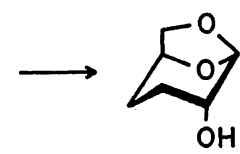

9

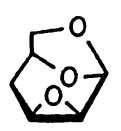

10

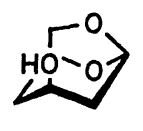

11

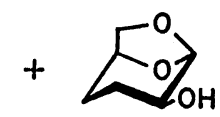

12
In this connection, it was reported that on lithium aluminum hydride reduction, 1,6:2,3-dianhydro-4deoxy- $\beta$-D-ribo-hexopyranose (corresponding to 8) exclusively afforded 1,6-anhydro-3,4-dideoxy- $\beta$-Derythro-hexopyranose (corresponding to 9), while 1,6 : 2,3-dianhydro-4-deoxy- $\beta$-D-lyxo-hexopyranose (corresponding to 10 ) gave a mixture of $\quad 1,6$-anhydro-2,4-dideoxy- $\beta$-D-threo-hexopyranose (corresponding to 11) and 1,6-anhydro3,4-dideoxy- $\beta$-D-threo-hexopyranose (corresponding to 12) in a ratio of $62.5: 37.5 .^{18}$ Therefore, the reaction product 9 in the lithium aluminum hydride reduction in the present monomer synthesis might be contaminated with a small amount of 11 and 12 (at most a few percent). In fact, unidentified signals, although of very weak intensities, were observed in the ${ }^{13} \mathrm{C}$ NMR spectrum of the product after being purified by sublimation. However, subsequent benzylation followed by purification by column chromatography provided pure 4(a)-benzyloxy-6,8-dioxabicyclo[3.2.1]octane (1) as judged from a single spot in thin layer chromatogram, a single peak in gas chromatogram, and a single set of ten signals in ${ }^{13} \mathrm{C}$ NMR spectrum.

\section{Polymerization of 4(a)-Benzyloxy-6,8-dioxabi-} cyclo[3.2.1]octane (1)

Polymerization of 1 was carried out in methylene chloride at different temperatures between -78 and $0^{\circ} \mathrm{C}$. Antimony pentafluoride, antimony pentachloride, trifluoromethanesulfonic acid, and boron trifluoride etherate were used as initiators. Table I summarizes some of the results of the polymerization of $\mathbf{1}$.

It is noteworthy that boron trifluoride etherate which acts as an effective initiator for stereospecific polymerization of the parent bicyclic acetal, 6,8dioxabicyclo[3.2.1] octane $^{19,20}$ was practically ineffective in initiating the polymerization of 1 under

Table I. Polymerization of $4(a)$-benzyloxy-6,8-dioxabicyclo[3.2.1]octane (1) ${ }^{\mathrm{a}}$

\begin{tabular}{|c|c|c|c|c|c|c|}
\hline \multicolumn{2}{|c|}{ Initiator } & \multirow{2}{*}{$\frac{\text { Temp }}{{ }^{\circ} \mathrm{C}}$} & \multirow{2}{*}{$\frac{\text { Time }}{\text { day }}$} & \multirow{2}{*}{$\frac{\text { Yield }^{\mathrm{b}}}{\%}$} & \multirow{2}{*}{$M_{n} \times 10^{-3 \mathrm{c}}$} & \multirow{2}{*}{$\frac{\alpha \text {-Content }}{\%}$} \\
\hline & $\mathrm{mmol}$ & & & & & \\
\hline $\mathrm{SbF}_{5}$ & 0.25 & 0 & 2 & $46^{e}$ & 6.2 & 56 \\
\hline $\mathrm{SbF}_{5}$ & 0.25 & -30 & 2 & 64 & 4.6 & 85 \\
\hline $\mathrm{SbF}_{5}$ & 0.25 & -60 & 2 & 40 & 6.7 & $\sim 100$ \\
\hline $\mathrm{SbF}_{5}$ & 0.25 & -60 & 5 & 71 & 9.9 & $\sim 100$ \\
\hline $\mathrm{SbF}_{5}$ & 0.25 & -60 & 7 & 80 & 10.9 & $\sim 100$ \\
\hline $\mathrm{SbF}_{5}$ & 0.10 & -60 & 5 & $33^{f}$ & 11.2 & $\sim 100$ \\
\hline $\mathrm{SbF}_{5}$ & 0.25 & -78 & 7 & 31 & 13.5 & $\sim 100$ \\
\hline $\mathrm{SbCl}_{5}$ & 0.25 & -78 & 7 & 54 & 11.0 & $\sim 100$ \\
\hline $\mathrm{CF}_{3} \mathrm{SO}_{3} \mathrm{H}$ & $0.63^{g}$ & -78 & 7 & 80 & 9.6 & $\sim 100$ \\
\hline $\mathrm{BF}_{3} \mathrm{OEt}_{2}$ & 0.25 & -60 & 7 & 2 & 3.6 & $\sim 100$ \\
\hline $\mathrm{BF}_{3} \mathrm{OEt}_{2}$ & 0.25 & -78 & 7 & trace & - & - \\
\hline
\end{tabular}

a Monomer, $5 \mathrm{mmol}$; solvent, $\mathrm{CH}_{2} \mathrm{Cl}_{2}, 1.0 \mathrm{ml}$.

b Methanol-insoluble polymer.

c By vapor pressure osmometry in benzene.

d By ${ }^{13}$ C NMR spectroscopy.

e Besides methanol-insoluble polymer, methanol-soluble oligomeric products were obtained. Yield, $15 \% ; M_{n}$, $1.2 \times 10^{3}$.

${ }^{f}$ Elemental analysis. Calcd for $\left(\mathrm{C}_{13} \mathrm{H}_{16} \mathrm{O}_{3}\right)_{n}$ : C, 70.89\%; H, 7.33\%. Found: C, $70.90 \%$; $\mathrm{H}, 7.25 \%$.

g Monomer, $12.5 \mathrm{mmol}$; solvent, $\mathrm{CH}_{2} \mathrm{Cl}_{2}, 3.3 \mathrm{ml}$. 
similar reaction conditions. Stronger acids such as antimony pentahalides and trifluoromethanesulfonic acid were required to obtain polymer 2 in moderate yield. The apparent reduced polymerizability of 1 compared with its parent compound is presumably due to increased activation enthalpy in the propagation caused by steric hindrance of the axially substituted bulky benzyloxy group in a terminal unit toward the approach of an incoming monomer.

On the other hand, the axially substituted benzyloxy group of $\mathbf{1}$ gives rise to a non-bonded strain in the monomeric state, which on polymerization can be relieved because of the equatorial oreintation of the benzyloxy group in the resulting polymer. This factor should enhance the thermodynamical polymerizability of $\mathbf{1}$ compared with that of the unsubstituted parent compound. However, this factor is not so dominative as to offset the opposed effect due to steric hindrance encountered in the propagation as mentioned above.

Recently, the reactivities of some bicyclic acetals in the acid-catalyzed hydrolysis were evaluated and correlated qualitatively with their reactivities in

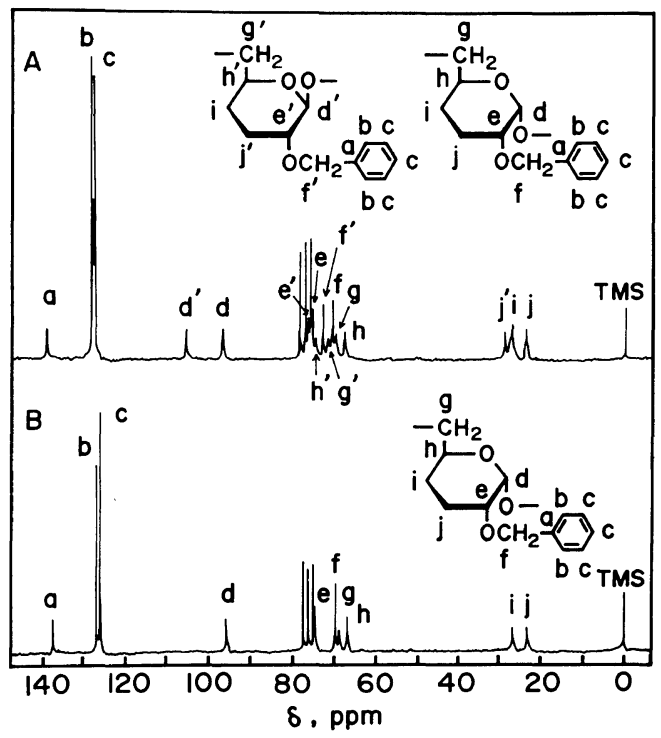

Figure 1. ${ }^{13} \mathrm{C}$ NMR spectra of poly(3-benzyloxytetrahydropyran-6,2-diyloxymethylene) (2): Solvent, $\mathrm{CDCl}_{3}$; temp, $50^{\circ} \mathrm{C}$; $25 \mathrm{MHz}$; internal reference, TMS. Polymerization conditions: A, solvent, $\mathrm{CH}_{2} \mathrm{Cl}_{2}$; initiator, $\mathrm{SbF}_{5}, 5 \mathrm{~mol} \%$; temp, $0^{\circ} \mathrm{C}$; time 2 days. $\mathrm{B}$, solvent, $\mathrm{CH}_{2} \mathrm{Cl}_{2}$; initiator, $\mathrm{SbF}_{5}, 5 \mathrm{~mol} \%$; temp, $-78^{\circ} \mathrm{C}$; time 3 days. cationic ring-opening polymerization. ${ }^{21,22}$ Therefore, the acid-catalyzed solvolysis of $\mathbf{1}$ was attempted at an ambient temperature in a mixed solvent of acetone- $d_{6}$ and deuterium oxide (volume ratio $3: 1$ ) in the presence of dichloroacetic acid as catalyst using ${ }^{1} \mathrm{H}$ NMR technique. ${ }^{21}$ However, 1 was not hydrolyzed at a measurable rate under the conditions where 6,8-dioxabicyclo[3.2.1] octane was hydrolyzed at a rate of $5.0 \times 10^{-6} \mathrm{~s}^{-1} .^{22}$ This finding is consistent with the aforementioned reduced reactivity of $\mathbf{1}$ compared with that of the unsubstituted parent compound.

Figure 1 shows the ${ }^{13} \mathrm{C}$ NMR spectra of the polymers obtained at $0^{\circ} \mathrm{C}(\mathrm{A})$ and $-78^{\circ} \mathrm{C}$ (B) using antimony pentafluoride as the initiator. The assignment of the signals was made by comparison with ${ }^{13} \mathrm{C}$ NMR spectrum of relevant polysaccharide analogues of different stereoregularities ${ }^{11,15,20}$ as well as by off-resonance technique. There appears only a set of ten sharp signals in the spectrum B, indicating that the polymer obtaind at $-78^{\circ} \mathrm{C}$ consists entirely of a structural unit ( $\alpha$-form) in which the exocyclic acetal oxygen is axially oriented to the tetrahydropyran ring. In contrast, the spectrum $\mathrm{A}$ of the polymer obtained at $0^{\circ} \mathrm{C}$ is rather complicated due to the coexistence of both " $\alpha$ form" and " $\beta$-form" units. The latter is a structural unit in which the exocyclic acetal oxygen is equatorially oriented to the tetrahydropyran ring. The " $\alpha$-form" content of the polymer can be estimated from the relative peak areas of the acetal carbon signals $d$ and $d^{\prime}$ ( $\delta 96.95$ and $\left.105.53 \mathrm{ppm}\right)$. As listed in Table I, the " $\alpha$-form" content of the polymer increased with a decrease in the polymerization temperature. The polymers prepared at $-60^{\circ} \mathrm{C}$ or below almost entirely consisted of the " $\alpha$-form" units. The formation of the " $\beta$-form" unit at higher temperatures is interpreted as participation of an oxacarbenium ion in propagation. ${ }^{19}$ The structure of the polymer was not affected by the type of initiator employed.

It should be noted here that the polymers of 6,8 dioxabicyclo[3.2.1]octane and its 4-bromo derivative showed ${ }^{13} \mathrm{C}$ NMR spectra in which some of the signals were split due to the dyad tacticities of the D,L-enantiomer units in the polymer chains..$^{20,23}$ In contrast, such splitting was not observed in the ${ }^{13} \mathrm{C}$ NMR spectra of the polymer of 1 , even in the expanded spectra covering the acetal and ether carbon regions in two different solvents (chloro- 


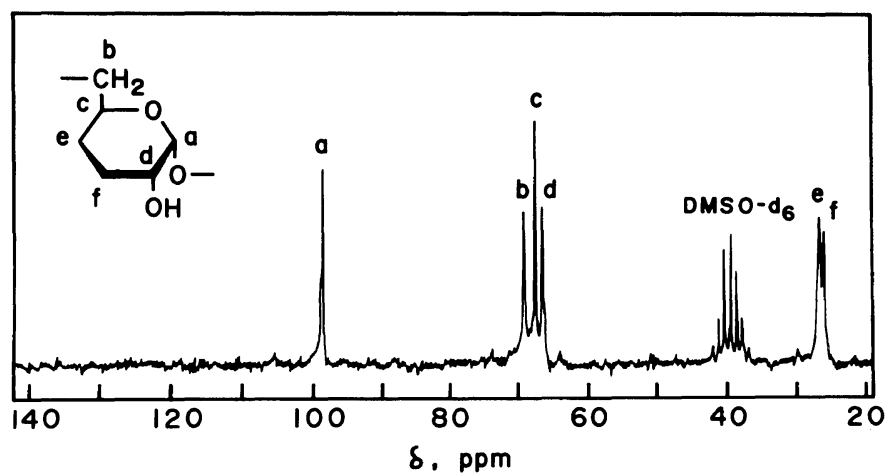

Figure 2. ${ }^{13} \mathrm{C}$ NMR spectrum of 3,4-dideoxy-(1 $\left.\rightarrow 6\right)-\alpha$-DL-erythro-hexopyranan (3): Solvent, DMSO- $d_{6}$; temp, $50^{\circ} \mathrm{C} ; 25 \mathrm{MHz}$; internal reference, TMS.

form- $d_{1}$ and benzene- $d_{6}$ ).

The polymers of 1 thus obtained were white powdery materials with a melting point of 60 $75^{\circ} \mathrm{C}$. They were soluble in benzene, carbon tetrachloride, chloroform, dioxane, and tetrahydrofuran, and insoluble in acetone, dimethyl sulfoxide, ethyl acetate, and $n$-hexane. The elemental analytical data of the polymer was satisfactory (Table I).

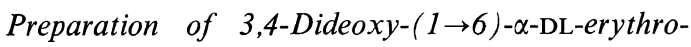
hexopyranan (3)

Debenzylation of polymer 2 by the conventional method using sodium metal in liquid ammonia gave a water-insoluble polymer along with a small amount of a water-soluble polymer. ${ }^{13} \mathrm{C}$ NMR spectroscopy and molecular weight determination revealed that the water soluble fraction was a low molecular weight oligomer $\left(\mathrm{DP}_{n} \doteqdot 6\right)$ having a structure identical with that of the water-insoluble fraction. ${ }^{1} \mathrm{H}$ and ${ }^{13} \mathrm{C}$ NMR spectroscopy confirmed that complete debenzylation occurred.

The ${ }^{13} \mathrm{C}$ NMR spectrum of debenzylated polymer 3 in dimethyl sulfoxide- $d_{6}$ solution is presented in Figure 2. There appear six relatively sharp signals whose assignments are shown in the figure. The ${ }^{13} \mathrm{C}$ NMR chemical shift data of $\mathbf{3}$ measured in dimethyl sulfoxide- $d_{6}$ and methanol- $d_{4}$ are presented in Table II.

Neither the ${ }^{13} \mathrm{C}$ NMR signals of 3 in dimethyl sulfoxide- $d_{6}$ nor those in methanol- $d_{4}$ showed splitting due to the tacticity of the polymer as described above. However, it cannot be decided at present whether this means undetectably small differences in chemical shifts between the isotactic and syndiotactic dyad placements of the D,L-enantiomer
Table II. ${ }^{13} \mathrm{C}$ NMR chemical shift data of 3,4-dideoxy- $(1 \rightarrow 6)$ - $\alpha$-DL-erythrohexopyranan (3) ${ }^{\mathrm{a}}$

\begin{tabular}{lllllll}
\hline \multirow{2}{*}{ Solvent } & \multicolumn{6}{c}{ Assignment } \\
\cline { 2 - 7 } & $\mathrm{C}(1)$ & $\mathrm{C}(2)$ & $\mathrm{C}(3)$ & $\mathrm{C}(4)$ & $\mathrm{C}(5)$ & $\mathrm{C}(6)$ \\
\hline $\mathrm{CD}_{3} \mathrm{SOCD}_{3}$ & 98.08 & 66.54 & 26.31 & 26.78 & 67.55 & 69.08 \\
$\mathrm{CD}_{3} \mathrm{OD}$ & 99.56 & 68.53 & 27.71 & 28.14 & 69.58 & 70.56
\end{tabular}

a Temp, $50^{\circ} \mathrm{C} ; 25 \mathrm{MHz}$. Chemical shift values are given in ppm from internal reference, tetramethylsilane. The carbon atoms are numbered according to the nomenclature in carbohydrate chemistry.

units $^{20}$ or the formation of a highly stereoregular polymer with long isotactic or syndiotactic sequences.

The synthetic polysaccharide 3 named 3,4dideoxy-( $1 \rightarrow 6)$ - $\alpha$-DL-erythro-hexopyranan according to the nomeclature of carbohydrate chemistry was soluble in methanol, dimethyl sulfoxide, and $N, N$-dimethylformamide, and insoluble in other common organic solvents and water. It melted at $130-140^{\circ} \mathrm{C}$ and decomposed in air at $280-300^{\circ} \mathrm{C}$.

The number average molecular weight of 3 determined by vapor pressure osmometry on solutions in $N, N$-dimethylformamide ranged from 4300 to 7800 . Elemental analytical data were in good agreement with the calculated values (Calcd for $\left(\mathrm{C}_{6} \mathrm{H}_{10} \mathrm{O}_{3}\right)_{n}: \mathrm{C}, 55.39 \% ; \mathrm{H}, 7.75 \%$. Found: $\mathrm{C}$, $55.27 \% ; \mathrm{H}, 7.87 \%$ ).

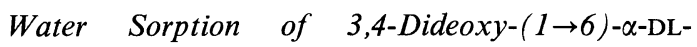
erythro-hexopyranan (3)

The water sorption of the synthetic poly- 


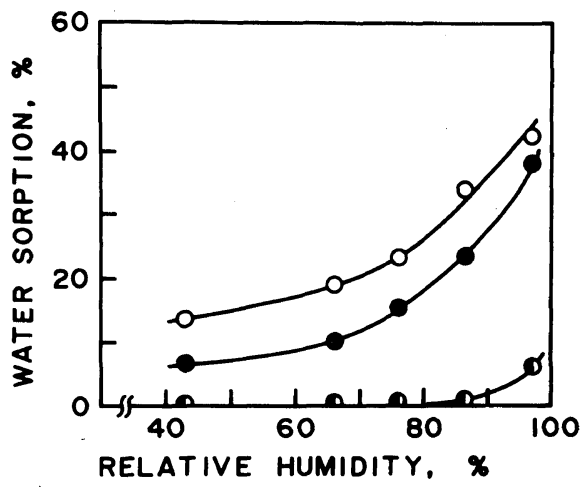

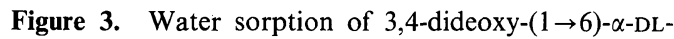
erythro-hexopyranan (3) and its related polymers at $25^{\circ} \mathrm{C}: \bigcirc$, dextran, $M_{n}=2.8 \times 10^{4} ; \bigcirc, 3, M_{n}=7.8 \times 10^{3}$; D, 2, $M_{n}=1.1 \times 10^{4}$.

saccharide 3 was measured at different relative humidities ranging from 43 to $97 \%$ at $25^{\circ} \mathrm{C}$, and compared with those of natural dextran and the benzylated polymer 2 . The water sorption as determined by the weight increase based on the dry samples became constant in seven to ten days up to $87 \%$ relative humidity. At $97 \%$ relative humidity, the weights of natural dextran and the synthetic polysaccharide 3 continued to increase even after 30 days. Therefore, water sorption at $97 \%$ relative humidity was tentatively determined on the basis of weight increase of the samples after exposure to moisture for 31 days. The results are graphically represented in Figure 3.

Although synthetic polysaccharide 3 possesses only one hydroxyl group in its repeating unit, its water sorption at a given relative humidity up to $87 \%$ amounts to $50-70 \%$ of that of natural dextran having a similar backbone structure with three hydroxyl groups per repeating unit. At $97 \%$ relative humidity, the water sorption of the synthetic polysaccharide 3 considerably approaches the water sorption of dextran. The relatively high water sorption of the synthetic polysaccharide 3 seems to arise, at least partly, from its structure consisting of D,L-enantiomeric monomer units; that is, the less ordered structure compared with the structure of dextran makes water molecules readily accessible to the hydrophilic portions of the polymer chain. The observed smaller difference in water sorption between synthetic polysaccharide $\mathbf{3}$ and dextran at
$97 \%$ relative humidity may be interpretable in terms of crystallization of the latter as previously demonstrated by Taylor et al. ${ }^{24,25}$ The benzylated polymer 2 hardly sorbed water except at $97 \%$ relative humidity.

\section{REFERENCES}

1. I. J. Goldstein and T. L. Hullar, Adv. Carbohydr. Chem., 21, 431 (1966).

2. C. Schuerch, Adv. Polym. Sci., 10, 173 (1972).

3. C. Schuerch, Acc. Chem. Res., 6, 184 (1973).

4. H. Sumitomo and M. Okada, Adv. Polym. Sci., 28, 47 (1978).

5. C. Schuerch, Adv. Carbohydr. Chem. Biochem., 39, 157 (1981).

6. M. Okada, H. Sumitomo, and H. Komada, Makromol. Chem., 179, 949 (1978).

7. H. Komada, M. Okada, and H. Sumitomo, Makromol. Chem., 179, 2859 (1978).

8. M. Okada, H. Sumitomo, M. Hasegawa, and H. Komada, Makromol. Chem., 180, 813 (1979).

9. H. Komada, M. Okada, and H. Sumitomo, Makromol. Chem., 181, 2305 (1980).

10. H. Komada, M. Okada, and H. Sumitomo, Macromolecules, 12, 5 (1979).

11. M. Okada, H. Sumitomo, and A. Sumi, Polym. Bull., 7, 431 (1982).

12. F. Sweet and R. K. Brown, Can. J. Chem., 46, 2289 (1968).

13. R. M. Strivastava and R. K. Brown, Can. J. Chem., 48, 830 (1970).

14. T. P. Murray, U. P. Singh, and R. K. Brown, Can. J. Chem., 49, 2132 (1972).

15. M. Okada, H. Sumitomo, and A. Sumi, Polym. J., 14, 59 (1982).

16. F. E. M. O’Brien, J. Sci. Inst., 25, 73 (1948).

17. M. Černý and J. Stanek, Jr., Adv. Carbohydr. Chem. Biochem., 34, 24 (1977).

18. J. Halbych, T. Trnka, and M. Černý, Collect. Czechoslov. Chem. Commun., 38, 2151 (1973).

19. M. Okada, H. Sumitomo, and Y. Hibino, Polym. J., 6, 256 (1974).

20. M. Okada, H. Sumitomo, and H. Komada, Macromolecules, 12, 395 (1979).

21. H. K. Hall, Jr., F. DeBlauwe, L. J. Carr, V. S. Rao, and G. S. Reddy, J. Polym. Sci., Polym. Symp., No. 56, 101 (1976).

22. M. Okada, H. Sumitomo, M. Kanie, and H. Komada, Makromol. Chem., 181, 2315 (1980).

23. M. Okada, H. Sumitomo, and A. Sumi, Macromolecules, 15, No. 5 (1982).

24. N. W. Taylor, H. F. Zobel, N. N. Hellman, and F. R. Senti, J. Phys. Chem., 63, 599 (1959).

25. N. W. Taylor, J. E. Chuskey, and F. R. Senti, J. Phys. Chem., 65, 1810 (1961). 